\title{
Formación del profesorado de Secundaria en España: Un estudio a través de los Másteres Oficiales en Educación Secundaria en universidades públicas
}

\author{
Francisco GONZÁLEZ SALA \\ Mar BISQUERT BOVERT \\ Julia HABA-OSCA \\ Julia OSCA-LLUCH
}

Datos de contacto:
Francisco González Sala
Universidad de Valencia
Francisco.Gonzalez-Sala@uv.es
Mar Bisquert Bovert
Universidad de Valencia
2amarbisquert@gmail.com
Julia Haba-Osca
Universidad de Valencia
Julia.Haba@uv.es
Julia Osca-Lluch
Universidad Politécnica de Valencia
juosllu@ingenio.upv.es

Francisco González Sala Universidad de Valencia

Mar Bisquert Bovert

Universidad de Valencia

Julia Haba-Osca

Universidad de Valencia

juosllu@ingenio.upv.es

\section{RESUMEN}

Es en el curso académico 2009-2010 cuando comienza a impartirse el Máster de Profesor de Educación Secundaria Obligatoria y Bachillerato, Formación Profesional, Enseñanza de Idiomas y Enseñanzas Deportivas. El objetivo del estudio es determinar las similitudes y diferencias en el plan de estudios del máster de formación del profesorado en universidades públicas españolas. Según el Registro de Universidades, Centros y Títulos se localizaron 43 universidades públicas en las que se imparte el Máster. Los resultados muestran una gran variedad de denominaciones con respecto al título del máster. Si bien presentan una estructura similar con respecto a las materias en los módulos genéricos y específicos, existen diferencias en cuanto a los créditos para cada una de estas. Son las especialidades de Educación Secundaria las más impartidas frente a las de Formación Profesional. La oferta es de más de 12.290 plazas, habiendo diferencias en cuanto al precio por crédito según el máster. Se puede concluir que el máster presenta una estructura muy similar acorde a la ley en la mayoría de las Universidades, detectándose la necesidad de una mayor y más amplia formación dirigida a la Formación Profesional. Tras diez años de impartición del máster cabe reflexionar acerca del mismo en relación a aspectos como las competencias del mismo, créditos pedagógico-didácticos, prácticum, profesorado que imparte el master o criterios de acceso al mismo.

PALABRAS CLAVE: Formación del profesorado, Educación Secundaria, Formación Profesional, Másteres oficiales, Universidades públicas, España. 


\title{
Secondary teacher training in spain: A study through the oficial Masters in Secondary Education in public universities
}

\begin{abstract}
It is during the academic year 2009-2010 when the Master's on Secondary Education, Vocational Training, Language Teaching and Sports Education started to be taught. The objective of this study is to determine the similarities and differences in the master's in teacher training degree curriculum in Spanish public universities. According to the Register of Universities, Centers and Titles there were 43 public universities located in which the Master is taught. The results show a great variety of denominations regarding the master's degree title. Although they present a similar structure with respect to the subjects in the generic and specific modules, there are differences in the credits assigned for each of these. Secondary education specialties are the most taught versus vocational training. The offer is for more than 12,290 graduates, having differences in the price per credit according to the master. It can be concluded that the master presents a very similar structure according to the law in most of Universities, detecting the need for greater and wider teaching at vocational training. After ten years of teaching this master's degree, it is necessary to reflect on it in relation to different criteria and aspects such as: competencies, pedagogical-didactic credits, practicum, teaching staff that teaches the master or the access.
\end{abstract}

KEYWORDS: Teacher training; Secondary Education; Vocational Training; Public universities; Oficial masters; Spain

\section{Introducción}

Tal y como apuntaba Vilches y Gil Pérez (2010) el Máster en Formación del profesorado en Secundaria supuso dar respuesta a una renovación de la enseñanza en España. La importancia del Máster fue recogida en reflexiones a través de diferentes monográficos de revistas de gran impacto, como fue el monográfico de la Revista de Educación en el año 2009.

Será en el curso 2009-2010 cuando comienza a impartirse el Master Universitario en Formación del Profesorado en Secundaria sustituyendo al antiguo curso de Certificación de Adaptación Pedagógica (CAP). El cursar y superar el Máster de Profesor de Educación Secundaria Obligatoria y Bachillerato, Formación Profesional, Enseñanza de Idiomas y Enseñanzas Deportivas (MUFP a partir de ahora), habilita 
para el ejercicio de la docencia como profesor en estos niveles educativos, siendo una condición necesaria de cara a participar en los procedimientos selectivos de ingreso y acceso a los Cuerpos de Profesores en estos niveles educativos.

La normativa que regula este Máster, Orden ECI/3858/2007, recoge la estructura del mismo en tres módulos, uno genérico, con un máximo de 12 créditos distribuidos en tres materias, Aprendizaje y desarrollo de la personalidad, Procesos y contextos educativos y Sociedad, familia y educación, otro específico de hasta 24 créditos, el cual incluye tres materias, Complementos para la formación disciplinar, Aprendizaje y enseñanza de las materias correspondientes e Innovación docente e iniciación a la investigación educativa, y un tercer módulo, Prácticum de 16 créditos, el cual incluye además de las prácticas, el Trabajo de fin de máster, siendo las comisiones académicas de cada máster las encargadas de estipular las correspondientes asignaturas en cada uno de los módulos.

No cabe duda que la formación tiene una influencia sobre el desarrollo de la profesión docente (Rebolledo, 2015) y sobre los estudiantes (Coelho, Oller y Serra, 2011), lo cual obliga a ir mejorando de forma permanente ésta. En este sentido, en la Conferencia de Decanos/as de Educación (2017) se recogen toda una serie de conclusiones dirigidas a la mejora del Máster en Formación del Profesorado en Secundaria.

El objetivo del presente estudio es determinar las similitudes y diferencias en relación a la formación del profesorado de Educación Secundaria Obligatoria y Bachillerato, Formación Profesional, Enseñanzas de Idiomas, Artísticas y Deportivas a través de los diferentes másteres oficiales impartidos en universidad públicas españolas en el curso académico 2018-2019.

\section{Método}

\section{Materiales y procedimiento}

Se seleccionaron aquellos másteres oficiales relacionados con la formación del profesorado en Educación Secundaria, Formación Profesional, Escuelas Oficiales de Idiomas y Enseñanzas Deportivas que se imparten en el curso 2018-2019 a partir del Registro de Universidades, Centros y Titulaciones (RUCT) del Ministerio de Educación y que fueran impartidos en universidades públicas, descartando los que eran impartidos por universidades privadas, con la excepción del máster de la Universitat Oberta de Catalunya, la cual 
comparte el máster con universidades públicas. Tras la selección de los másteres se pasó a consultar los planes de estudios publicados en el Boletín Oficial del Estado según aparece en el RUCT y en las páginas oficiales de cada máster, seleccionando la información relevante para el presente estudio. En los casos en los que la información recogida en el BOE y en la web oficial del Máster era diferente se decidió tomar información de la página web.

Con respecto a las especialidades se recogieron aquellas que eran impartidas en el curso académico 2018-2019, descartando las que no fueron impartidas a pesar de estar recogidas en los planes de estudios según BOE. Además, para el presente estudio se descartó la especialidad de Orientación Educativa, al presentar características diferenciales con respecto al resto de especialidades.

Las variables de estudio que se recogieron fueron: Créditos totales (CT), Créditos obligatorios comunes (COC) referentes al módulo genérico, Créditos obligatorios/optativos referentes al módulo específico (COE), Créditos optativos (CO) considerando estos como aquellos créditos ofertados por encima de los 60 créditos necesarios para la obtención del título, Créditos de TFM (CTFM), Créditos de practicum (CP), Materias relacionadas con el módulo genérico y específico, Especialidades ofertadas y número de másteres que la ofrecen, Número de plazas ofertadas, Duración del máster y Modalidad presencial o semipresencial.

\section{Resultados}

El número total de másteres seleccionados fueron 43, todos ellos pertenecientes a la rama de las ciencias jurídicas y sociales, con una duración total de 60 créditos.

\section{MUFP denominación y niveles educativos}

Con respecto a la denominación de los másteres, estos se pueden agrupar en diferentes categorías según el título del máster:

Másteres que incluyen en el título Educación Secundaria Obligatoria, Bachillerato, Formación Profesional y Enseñanzas de Idiomas, recogido en 34 másteres, como son los impartidos en las universidades Pompeu Fabra (UPF), Oberta de Catalunya (UOC), Autónoma de Barcelona (UAB), Universidad de Almería (UAL), Universidad de Alicante (UA), Universidad de Alcalá (UAH), Universidad de Barcelona (UB), Universidad de Burgos (UBU), Universidad de Cádiz (UCA), Universidad de Castilla-La Mancha (UCLM), Universidad 
Complutense de Madrid (UCM), Universidad de Córdoba (UCO), Universidad de Girona (UDG), Universidad de Granada (UGR), Universidad de Huelva (UHU), Universidad de Jaén (UJAEN), Universidad de La Rioja (UNIRIOJA), Universidad de León (UNILEON), Universidad de Lleida (UdL), Universidad de Málaga (UMA), Universidad de Salamanca (USAL), Universidad de Santiago de Compostela (USC), Universidad de Sevilla (US), Universidad de Valladolid (UVA), Universidad de Vigo (UVIGO), Universidad Euskal Herria (EHU), Universitat Jaume I de Castelló (UJI), Universidad Miguel Hernández de Elche (UMH), Universidad Nacional de Educación a Distancia (UNED), Universidad Pablo de Olavide (UPO), Universidad Politècnica de Catalunya (UPC), Universidad Rey Juan Carlos (URJC), Universidad Rovira i Virgili (URV) y Universidad de A Coruña (UAC).

Otros másteres además incluyen Enseñanzas Artísticas, como el de la Universidad de Murcia (UM) y Enseñanza Deportiva como el de la Universidad de Zaragoza (UNIZAR).

Másteres que incluyen únicamente Educación Secundaria, Bachillerato y Formación Profesional, son el de la Universidad de Oviedo (UNIOVI) y la Universidad Politécnica de Madrid (UPM). En el caso de la Universidad Autónoma de Madrid (UAM) incluye Educación Secundaria y Bachillerato, y en las Universidades de Cantabria (UNICAN), Extremadura (UEx), Pública de Navarra (UPNA) y Valencia (UVEG) el título solo incluye la Enseñanza Secundaria Obligatoria.

\section{UFP caracteristicas generales}

A la hora de analizar la distribución de créditos según si estos son obligatorios o prácticos existen diferencias y similitudes entre unos másteres y otros. En relación a los créditos comunes obligatorios referidos al módulo genérico, estos van desde los 12 hasta los 44 créditos de la UPC, si bien es entre 12 y 15 créditos en este módulo lo más frecuente (ver tabla 1).

Los créditos específicos, considerados obligatorios $u$ optativos, comprenden entre los 12 créditos de la UMH y los 32 créditos de la UVA, si bien hay algunos másteres que no recogen créditos específicos como tal, como son los másteres de las universidades UPV/EHU, UJI, UPC, UVEG, USAL, UJAEN, UHU, UdG, UBU o la UAB, considerando estos como optativos no específicos. Teniendo en consideración la optatividad como la oferta de un mayor número de créditos a los necesarios para cursar el máster, solo 11 másteres ofertan éstos, como son los de las universidades de Alcalá de Henares, Almería, Cádiz, Córdoba, Granada, Jaén, León, Sevilla, Pablo Olavide, Málaga y Navarra (ver tabla 1). 
Al analizar los créditos de prácticum todos los títulos ofrecen este tipo de créditos, variando entre los 10 y los 18 créditos, diferenciándose el master de UNILEON que contaría con 8 créditos prácticos en centros más 2 créditos de seminarios relacionados con la preparación de las prácticas. Por su parte, los dirigidos al TFM son mayoritariamente 6 créditos, salvo para el master de UNIRIOJA que cuenta con 6,5 créditos.

Con respecto a la modalidad del máster, ésta suele ser mayoritariamente de naturaleza presencial, si bien en algunos títulos ésta es semipresencial u on-line, estipulando un porcentaje de créditos no presenciales, como ocurre en el máster de la UPV/EHU donde un $80 \%$ son presenciales.

El número mínimo de plazas ofertadas es de 12.290, habiendo un mayor número de plazas si se tiene en cuenta que en algunos másteres no se ha podido recoger este dato. Según el máster estas plazas van desde las 60 de la UdL a las 1.000 plazas de la UVEG, distribuyéndose después según las especialidades o itinerarios.

Es en el curso académico 2009-2010 cuando se ofrece la primera edición del máster, como así ocurre en la mayoría de los títulos. 
Tabla 1.

Caracteristicas generales de los Másteres Oficiales de Formación del Profesorado en la Universidad española

\begin{tabular}{|c|c|c|c|c|c|c|c|c|}
\hline Máster & $\mathrm{COC}$ & $\mathrm{CE}$ & COp & $\mathrm{CP}$ & Ctfm & Modalid. & $\begin{array}{l}\mathrm{N}^{0} . \\
\text { plazas }\end{array}$ & $\begin{array}{l}\text { Curso } \\
\text { implantació } \\
\text { n }\end{array}$ \\
\hline UPF-UOC $^{1}$ & 15 & 25 & 0 & 14 & 6 & SP/On-line & 110 & n.e. \\
\hline UAM & 14 & 26 & 0 & 14 & 6 & $\mathrm{P}$ & 230 & 2009-2010 \\
\hline UAB & 15 & 27 & 0 & 12 & 6 & $\mathrm{P}$ & 180 & n.e. \\
\hline UAC & 16 & 26 & 0 & 12 & 6 & $\mathrm{P}$ & 195 & $2009-2010$ \\
\hline UCM & 12 & 30 & 0 & 12 & 6 & $\mathrm{P}$ & 500 & $2009-2010$ \\
\hline UAH & 12 & 24 & $8(16)$ & 10 & 6 & $\mathrm{P}$ & 250 & $2009-2010$ \\
\hline UA & 14 & 30 & 0 & 10 & 6 & $\mathrm{P}$ & 500 & 2009-2010 \\
\hline UAL & 12 & 24 & $8(16)^{3}$ & 10 & 6 & $\mathrm{P}$ & n.e. & 2009-2010 \\
\hline UB & 15 & 25 & 0 & 14 & 6 & $\mathrm{P}$ & 390 & $2009-2010$ \\
\hline UBU & 17 & 24 & 0 & 11 & 8 & $\mathrm{P}$ & 200 & 2009-2010 \\
\hline UCA & 12 & 24 & $4(40)$ & 14 & 6 & $\mathrm{P}$ & 210 & 2013-2014 \\
\hline UNICAN & 15 & 24 & 0 & 15 & 6 & $\mathrm{P}$ & 120 & 2009-2010 \\
\hline UCLM & 12 & 24 & 0 & 18 & 6 & $\mathrm{P}$ & 425 & n.e. \\
\hline $\mathrm{UCO}$ & 12 & 24 & $8(16)$ & 10 & 6 & $\mathrm{P}$ & n.e. & $2009-2010$ \\
\hline UEx & 12 & 30 & 0 & 12 & 6 & $\mathrm{P}$ & 485 & n.e. \\
\hline UdG & 15 & 28 & 0 & 14 & 6 & Mixta & 120 & n.e. \\
\hline UGR & 12 & 24 & $8(20)$ & 10 & 6 & $\mathrm{P}$ & n.e. & 2009-2010 \\
\hline UHU & 12 & 24 & 0 & 18 & 6 & $\mathrm{P}$ & 210 & 2016-2017 \\
\hline UJAEN & $8 / 12^{2}$ & 24 & $8(16)$ & 10 & 6 & $\mathrm{P}$ & 300 & 2009-2010 \\
\hline UNIRIOJA & 13,5 & 27 & 0 & 13 & 6,5 & SP & 175 & $\begin{array}{l}2009-2010 \\
\text { SP 2017- } \\
2018\end{array}$ \\
\hline UNILEON & 12 & 24 & $8(16)^{4}$ & $8(2)$ & 6 & $\mathrm{P}$ & 180 & n.e. \\
\hline UdL & 15 & 25 & 0 & 14 & 6 & $\mathrm{P}$ & 60 & 2009-2010 \\
\hline UMA & 12 & $24+8^{5}$ & 0 & 10 & 6 & $\mathrm{P}$ & 550 & 2009-2010 \\
\hline UM & 15 & 27 & 0 & 12 & 6 & $\mathrm{P}$ & 410 & n.e. \\
\hline UNIOVI & 15 & $23+3^{6}$ & 0 & 13 & 6 & $\mathrm{P}$ & 190 & $2009-2010$ \\
\hline USAL & 15 & 27 & 0 & 12 & 6 & $\mathrm{P}$ & 300 & 2009-2010 \\
\hline USC & 16 & 26 & 0 & 12 & 6 & $\mathrm{P}$ & 240 & n.e. \\
\hline US & 12 & 24 & $8(72)$ & 10 & 6 & $\mathrm{P}$ & 383 & 2009-2010 \\
\hline UVA & 12 & 32 & 0 & 10 & 6 & $\mathrm{P}$ & 425 & n.e. \\
\hline UVIGO & 16 & 26 & 0 & 12 & 6 & $\mathrm{P}$ & 280 & n.e. \\
\hline UNIZAR & 18 & 26 & 0 & 10 & 6 & $\mathrm{P}$ & 568 & 2009-2010 \\
\hline UPV/EHU & 18 & 24 & 0 & 12 & 6 & $\mathrm{P}(80 \%)$ & 264 & n.e. \\
\hline UJI & 20 & 24 & 0 & 10 & 6 & $\mathrm{P}$ & 180 & n.e. \\
\hline $\mathrm{UMH}$ & 18 & 24 & 0 & 12 & 6 & $\mathrm{SP}$ & 400 & n.e. \\
\hline UNED & 15 & 27 & 0 & 12 & 6 & A distancia & 710 & n.e. \\
\hline UPO & 12 & 24 & $8(40)$ & 10 & 6 & $\mathrm{P}$ & 260 & $2009-2010$ \\
\hline UPC & 12 & 28 & 0 & 14 & 6 & $\mathrm{P}$ & 90 & n.e. \\
\hline
\end{tabular}




\begin{tabular}{lllllllll} 
UPM & 18 & 18 & $6(12)$ & 12 & 6 & $\mathrm{P}$ & 120 & n.e. \\
\hline UPNA & 14 & 27 & $3(6)$ & 10 & 6 & $\mathrm{P}$ & 120 & n.e. \\
\hline URJC & 12 & 24 & 0 & 12 & 6 & $\mathrm{P}$ & 535 & n.e. \\
\hline URV & 20 & 20 & 0 & 14 & 6 & $\mathrm{P}$ & 150 & $2009-2010$ \\
\hline UVEG & 16 & 28 & 0 & 10 & 6 & $\mathrm{P}$ & 1000 & $2009-2010$ \\
\hline $\begin{array}{l}\text { UAB, UB, } \\
\text { UOC, UPC } \\
\text { y UPF }\end{array}$ & 12 & 28 & 0 & 14 & 6 & $\mathrm{P}$ & 80 & $2013-2014$ \\
\hline
\end{tabular}

Nota: (1) Master impartido por ambas universidades (Pompeu Fabra es semipresencial. Especialidades Inglés y Ciencias de la naturaleza) y (Oberta de Catalunya es on line, Especialidades Formación y Orientación Laboral y Orientación Educativa). (2) en el caso de la Especialidad de Orientación educativa los créditos del módulo genérico son 12, mientras que en el resto de especialidades son 8 créditos). (3) en el caso del Máster de la UAL las asignaturas optativas son múltiples, si bien muchas de ellas son optativas/obligatorias relacionadas con las especialidades, habiendo 4 asignaturas con 4 créditos cada una de ellas que son optativas sin pertenecer a ninguna de las especialidades ofertadas. (4) El número de créditos optativos depende de la especialidad. (5) Tres créditos están relacionados con la participación en un ciclo de conferencias. (6) No especifica el número de créditos ofertados, si bien las asignaturas ofertadas varían en función de la especialidad).

\section{MUFP especialidades ofertadas}

En la tabla 2 se recogen las diferentes especialidades ofertadas en el curso académico 2018-2019 y las universidades en las que se imparte cada una de ellas. En algunos casos existe discrepancia entre lo que recoge el plan de estudios publicado en el BOE y lo publicado en la página oficial del máster en el último curso académico, por lo que se ha optado por coger la información de la página oficial.

En el caso concreto de las Universidades catalanas (UB, UAB, UOC, UPC, UdL, URV, UPF, UDG) la Generalitat de Catalunya distribuye las especialidades y plazas que se impartirán en cada una de sus universidades para el curso 2018-2019, según acuerdo de Junta del 29 de marzo de 2017. Esta distribución puede consultarse en el siguiente enlace: http://universitats.gencat.cat/web/.content/03_Que_estudiar_i_a_on/masters/ma ster_formacio_professorat/documents/especialitats-master-de-professorat.pdf

Con respecto a las especialidades, hay másteres donde éstas están relacionadas con materias o asignaturas concretas, como por ejemplo Física y Química, Biología y Geología o Matemáticas, mientras que en otros másteres se ofertan especialidades de carácter general, como por ejemplo Ciencias Experimentales, la cual engloba las materias anteriormente enumeradas. Algo similar sucede con las especialidades en Ciencias Sociales, Humanidades, Idiomas o Artes Plásticas y Visuales.

Son las especialidades relacionadas con la Educación Secundaria las mayormente representadas en los diferentes másteres, en concreto las especialidades de Biología y Geología, Física y Química y Matemáticas dentro de las ciencias experimentales, y Educación física, Inglés, Geografía e Historia y Lengua Castellana y Literatura. Con respecto a las especialidades relacionadas

212 ISSN 0213-8646 | E-ISSN 2530-3791 • Revista Interuniversitaria de Formación del Profesorado, 95 (34.2) (2020), 205-224 
con la Formación Profesional destaca la formación en Administración y Empresa, Formación y Orientación Laboral y Procesos Industriales.

Tabla 2.

Relación de especialidades ofertadas según los Másteres Oficiales de Formación del Profesorado por Universidades públicas en el curso académico 2018-2019.

\section{Biología y Geología}

$\mathbf{N}^{\mathbf{0}}$.

- Alcalá, Almería, Alicante, Autónoma de Barcelona, Autónoma de Madrid, 30

Barcelona, Burgos, Cádiz, Cantabria, Castilla-La Mancha, Complutense de

Madrid, Córdoba, Extremadura, Girona, Granada, Huelva, Jaén, León, Málaga,

Murcia, Oviedo, Pablo Olavide, Pública de Navarra, Rey Juan Carlos, Rovira i

Virgili, Salamanca, Sevilla, Valencia, Valladolid, Zaragoza.

\section{Física y Química}

- Alcalá, Alicante, Autónoma de Barcelona, Autónoma de Madrid, Barcelona,

Burgos, Cádiz, Castilla-La Mancha, Complutense de Madrid, Córdoba,

Extremadura, Girona, Granada, Huelva, Jaén, La Rioja, León, Málaga, Murcia,

Oviedo, Pablo Olavide, Politécnica de Madrid, Pública de Navarra, Rey Juan

Carlos, Rovira i Virgili, Salamanca, Sevilla, UNED, Valencia, Valladolid,

Zaragoza.

- En la Universidad de Cantabria la especialidad es (Física y Química y Tecnología)

Ciencias naturales (se imparte conjuntamente Biología, Geología, Física y Química)

- Miguel Hernández y Pompeu Fabra-Oberta de Catalunya.

- País Vasco (Biología, Geología, Física, Química y Matemáticas).

\section{Ciencias experimentales}

- $\quad$ A Coruña, Santiago de Compostela y Vigo (incluye Biología, Geología, Física y 4 Química).

- Jaume I (Física, Química, Ciencias de la naturaleza, Tecnología e Informática).

\section{Matemáticas}

- Alcalá, Alicante, Almería, Autónoma de Madrid, Burgos, Cádiz, Cantabria,

Castilla-La Mancha, Complutense de Madrid, Córdoba, Extremadura, Granada,

Jaén, Jaume I, La Rioja, León, Málaga, Miguel Hernández, Murcia, Oviedo,

Pablo de Olavide, Politécnica de Madrid, Rey Juan Carlos, Salamanca, Santiago

de Compostela, Sevilla, UNED, Valencia Valladolid y Zaragoza.

- $\quad$ Especialidad interuniversitaria (UAB, UB, UOC, UPC y UPF).

\section{Educación física y deportes}

- A Coruña, Alcalá, Alicante, Almería, Autónoma de Madrid, Barcelona,

Cádiz,Cantabria, Castilla La Mancha, Complutense de Madrid, Córdoba,

Extremadura, Granada, Huelva, Jaén, León, Lleida, Málaga, Miguel Hernández,

Murcia, Oviedo, Pablo de Olavide, País Vasco, Rey Juan Carlos, Salamanca,

Sevilla, UNED, Valencia Vigo y Zaragoza.

- $\quad$ Politécnica de Madrid (se imparte en INEF).

\section{Filosofía}

- Autónoma de Madrid, Barcelona, Complutense de Madrid, Granada, Murcia,

Oviedo, Salamanca, Santiago de Compostela, Sevilla, UNED, Valencia,

Valladolid y Zaragoza.

\section{Francés}


- Alicante, Autónoma de Barcelona, Autónoma de Madrid, Castilla-La Mancha,

Complutense de Madrid, Extremadura, Granada, Jaén, Murcia, Oviedo, Pablo de

Olavide, Pública de Navarra, Salamanca, Sevilla, UNED, Valencia, Valladolid y Zaragoza.

\section{Inglés}

Alcalá, Alicante, Almería, Autónoma de Barcelona, Autónoma de Madrid, 30

Barcelona, Burgos, Castilla-La Mancha, Complutense de Madrid, Córdoba, Extremadura, Granada, Huelva, Jaén, Jaume I, La Rioja, León, Lleida, Murcia, Oviedo, Pablo de Olavide, Pompeu Fabra-Oberta de Catalunya, Pública de Navarra, Rey Juan Carlos, Salamanca, Sevilla, UNED, Valencia, Valladolid y Zaragoza.

\begin{tabular}{|c|c|c|}
\hline \multicolumn{3}{|c|}{ Alemán } \\
\hline- & Complutense de Madrid, Sevilla, Valencia y Valladolid. & 4 \\
\hline \multicolumn{3}{|c|}{ Italiano } \\
\hline- & Complutense de Madrid, UNED, Sevilla. & 3 \\
\hline \multicolumn{3}{|c|}{ Portugués } \\
\hline- & Extremadura. & 1 \\
\hline \multicolumn{3}{|c|}{ Chino } \\
\hline- & Granada. & 1 \\
\hline \multicolumn{3}{|c|}{ Lenguas extranjeras (Alemán, Portugués, Inglés, Italiano, Francés) } \\
\hline- & $\begin{array}{l}\text { A Coruña (lenguas extranjeras), Cádiz (Inglés, Alemán, Francés), Málaga (Inglés } \\
\text { y Francés), Miguel Hernández (Inglés, Alemán, Francés, Italiano, Portugués, } \\
\text { Latín, Griego), Salamanca (Alemán, Italiano y Portugués), Santiago de } \\
\text { Compostela y Vigo. } \\
\text { En el caso del Máster de Barcelona y la Autónoma de Barcelona la especialidad } \\
\text { puede incluir Inglés, Francés y alguna otra lengua extranjera. }\end{array}$ & 9 \\
\hline
\end{tabular}

\section{Formación y Orientación Laboral}

- $\quad$ A Coruña, Alicante, Burgos, Complutense de Madrid, Córdoba, Granada, Jaén, 20 León, Málaga, Miguel Hernández, Oviedo, Pablo de Olavide, Pompeu FabraOberta de Catalunya, Rey Juan Carlos, Salamanca, Sevilla, UNED, Valencia, Valladolid y Vigo.

\section{Geografía e Historia (Historia del Arte)*}

- Alcalá, Alicante, Almería, Autónoma de Barcelona, Autónoma de Madrid,

Barcelona, Burgos, Castilla la Mancha*, Complutense de Madrid, Córdoba,

Extremadura, Girona, Granada, Huelva, Jaén, Jaume I, La Rioja, León, Murcia, Oviedo, Pablo de Olavide, Pública de Navarra, Rey Juan Carlos, Rovira i Virgili Salamanca, Santiago de Compostela, Sevilla, UNED, Valencia, Valladolid, Vigo y Zaragoza.

- Cantabria y Cádiz (incluyen Geografía, Historia y Filosofía).

\section{Ciencias sociales y/o humanidades}

- Málaga (Geografía, Historia y Filosofía). Miguel Hernández (Geografía, 5 Historia, Música y Filosofía). Navarra (incluye Geografía, Historia y Filosofía). País Vasco (Matemáticas, Economía, Filosofía, Geografía, Historia, Historia 
Contemporánea y moderna del País Vasco e Historia del Arte). Rey Juan Carlos (Geografía, Historia y Arte).

\section{Lengua Castellana y Literatura}

Alcalá, Alicante, Almería, Autónoma de Madrid, Burgos, Cádiz, Cantabria,

Castilla-La Mancha, Complutense de Madrid, Córdoba, Extremadura, Granada,

Huelva, Jaén, Jaume I, La Rioja, León, Murcia, Oviedo, Pablo de Olavide, Pública de Navarra, Rey Juan Carlos, Sevilla, UNED, Valencia, Valladolid y Zaragoza.

- En el caso del máster de Málaga incluye (Lengua castellana, literatura, Latín y Griego).

- Autónoma de Barcelona, Barcelona, Girona, Rovira i Virgili (incluye Catalán). País Vasco (incluye Euskera). Miguel Hernández (incluye Valenciano). Santiago de Compostela, Vigo, A Coruña (incluye Gallego).

\begin{tabular}{|c|c|c|}
\hline \multicolumn{3}{|c|}{ Otras lenguas oficiales } \\
\hline- & $\begin{array}{l}\text { Alicante (valenciano), Oviedo (asturiano), Pública de Navarra (euskera), } \\
\text { Valencia (valenciano). }\end{array}$ & 4 \\
\hline \multicolumn{3}{|c|}{ Orientación Educativa } \\
\hline- & $\begin{array}{l}\text { Alcalá, Alicante, Autónoma de Madrid, Burgos, Cádiz, Cantabria, Complutense } \\
\text { de Madrid, Córdoba, Extremadura, Granada, Huelva, Jaén, Málaga, Miguel } \\
\text { Hernández, Murcia, Oviedo, País Vasco, Pompeu Fabra-Oberta de Catalunya, } \\
\text { Rey Juan Carlos, Salamanca, Santiago de Compostela, Sevilla, UNED, Valencia, } \\
\text { Valladolid, Vigo y Zaragoza. }\end{array}$ & 27 \\
\hline \multicolumn{3}{|c|}{ Lenguas clásicas: Griego y/o Latín / Cultura clàssica } \\
\hline- & $\begin{array}{l}\text { Autónoma de Madrid, Barcelona, Complutense de Madrid, GranadaMurcia, } \\
\text { Oviedo, Salamanca, Santiago de Compostela, Sevilla, UNED, Valencia, } \\
\text { Valladolid y Zaragoza. }\end{array}$ & 13 \\
\hline \multicolumn{3}{|c|}{ Economía } \\
\hline & $\begin{array}{l}\text { Alicante, Castilla La ManchaLa Rioja, León, Oviedo, Rovira i Virgili, Valencia } \\
\text { y Valladolid. }\end{array}$ & 8 \\
\hline \multicolumn{3}{|c|}{$\begin{array}{c}\text { Ciencias Sociales (incluyendo materias como ADE, Economía, Comercio, Empresa, } \\
\text { Administración, Gestión laboral) }\end{array}$} \\
\hline- & $\begin{array}{l}\text { Almería, Almería, Autónoma de Madrid, Cádiz, Cantabria, Castilla La Mancha, } \\
\text { Complutense de Madrid, Córdoba, Extremadura, Granada, Jaén, Málaga, Miguel } \\
\text { Hernández, Murcia, Pablo Olavide, País Vasco, Pública de Navarra, Rey Juan } \\
\text { Carlos, Rovira i Virgili, Salamanca, Sevilla, UNED, Valencia, Vigo y Zaragoza. } \\
\text { Miguel Hernández (FP Rama Administrativa) }\end{array}$ & 27 \\
\hline \multicolumn{3}{|c|}{ Informática / Tecnología (como materias independientes) } \\
\hline & $\begin{array}{l}\text { A Coruña (T), Alcalá (T), Alicante (I, T), Burgos (T), Córdoba (I), Extremadura } \\
\text { (I, T), Jaén (I), La Rioja (T), León, Miguel Hernández (I, T), Murcia (T), Oviedo } \\
\text { (I, T), País Vasco (T) Politècnica de Catalunya (T), Politécnica de Madrid (T), } \\
\text { Pública de Navarra (T), Rey Juan Carlos (I, T) Rovira i Virgili (T), Salamanca } \\
\text { (T), Sevilla (I) y UNED (I). } \\
\text { Castilla La Mancha, Valladolid, Zaragoza, Rey Juan Carlos, Vigo, Complutense } \\
\text { de Madrid (la especialidad es Informática y Tecnología como un único } \\
\text { itinerario). }\end{array}$ & 27 \\
\hline
\end{tabular}




\section{Tecnología y Procesos Industriales (Ciclos Formativos Industriales)}

- Almería, Castilla La Mancha, Córdoba, Jaén, León, Politècnica de Catalunya, 16

Rovira i Virgili, Sevilla y Zaragoza

- Huelva, Cádiz, Granada y Málaga la especialidad se denomina (Tecnología, Informática y Procesos Industriales), Murcia (Industriales e Informática), UNED (Tecnología de Máquinas, Fluidos y Mantenimiento), Valladolid (Tecnología Agraria, Alimentaria y Forestal).

\section{Dibujo (Técnico/Artístico/Diseño)/Expresión Gráfica/Comunicación Audiovisual}

- $\quad$ Autónoma de Madrid, Barcelona, Oviedo, Rey Juan Carlos, Salamanca (Dibujo 9 y Comunicación audiovisual como especialidades diferentes), Valencia y Vigo.

- País Vasco (Educación Artística) y Politécnica de Madrid (Expresión gráfica).

Materias incluidas dentro de un mismo módulo como Dibujo, Imagen y Artes

Plásticas, Expresión artística, Educación artística, Imagen, Artes plásticas y visuales

- A Coruña, Alcalá, Almería, Cádiz, Cantabria, Complutense de Madrid, Córdoba,

Granada, Jaén, Málaga, Miguel Hernández, Murcia, Santiago de Compostela,

Sevilla y Zaragoza.

- Castilla La Mancha y Extremadura (Artes).

- País Vasco (Educación Musical, Danza y Artes Escénicas).

- Zaragoza (Música y Danza).

\section{Música}

- Alicante, Autónoma de Madrid, Cantabria, Complutense de Madrid, Córdoba,

Granada, Jaén, Málaga, Murcia, Oviedo, Salamanca, Santiago de Compostela, Sevilla, Valencia y Valladolid.

\begin{tabular}{|c|c|c|}
\hline \multicolumn{3}{|c|}{ Hostelería y Turismo } \\
\hline- & Córdoba, Granada, Jaén, Málaga, Murcia, Rey Juan Carlos y Sevilla. & 7 \\
\hline \multicolumn{3}{|c|}{ Construcciones civiles, edificación y dibujo } \\
\hline & Alicante & 1 \\
\hline \multicolumn{3}{|c|}{$\begin{array}{l}\text { Intervención sociocomunitaria o FP Especialidad Servicios Socioculturales y a la } \\
\text { Comunidad }\end{array}$} \\
\hline & $\begin{array}{l}\text { Burgos, Extremadura, León, Rey Juan Carlos, UNED, Valencia y Valladolid } \\
\text { Miguel Hernández (Servicios sociosanitarios e intervención sociocomunitaria). }\end{array}$ & 8 \\
\hline \multicolumn{3}{|c|}{ FP Biosanitaria/Salud/Procesos Sanitarios, Ciencias de la Salud } \\
\hline & $\begin{array}{l}\text { Alicante, Barcelona, Cantabria, Córdoba, Extremadura, Granada, Jaén, Málaga, } \\
\text { Murcia, Salamanca, Sevilla y Valencia. }\end{array}$ & 12 \\
\hline \multicolumn{3}{|c|}{ Sector Servicios* y Sector primario y secundario** } \\
\hline & Santiago de Compostela, Vigo. & 2 \\
\hline \multicolumn{3}{|c|}{ Otras especialidades } \\
\hline $\begin{array}{l}\text { Alica } \\
\text { Form } \\
\text { Secto } \\
\text { Valer } \\
\text { Proce } \\
\text { Alime } \\
\text { Infor }\end{array}$ & $\begin{array}{l}\text { e (Ciclos Formativos - Profesorado de Educación Secundaria) y (Ciclos } \\
\text { ivos - Cuerpo profesorado técnico en Formación Profesional), Cantabria (FP } \\
\text { Primario, Industrial y Servicios), Miguel Hernández (FP para ciclos formativos), } \\
\text { la (FP Especialidades de Informática y Sistemas Electrónicos), Zaragoza (FP en } \\
\text { os Químicos, Sanitarios, de Imagen Personal, Agrarios, Marítimo-Pesqueros, } \\
\text { tarios y de Servicios a la Comunidad) y (Administración, Comercio, Hostelería, } \\
\text { ítica y Formación y Orientación Laboral para FP). }\end{array}$ & 5 \\
\hline
\end{tabular}




\section{MUFP distribución de créditos por módulo genérico o específico}

El módulo genérico está formado principalmente por una estructura de tres materias (ver tabla 3). Aprendizaje y desarrollo de la personalidad, impartida en 36 másteres, teniendo una duración entre 3 y 6 créditos, siendo lo más frecuente 4 créditos. Procesos y contextos educativos, presente en 37 másteres con una duración entre 3 y 8 créditos, siendo lo más frecuente 4 créditos. Sociedad, familia y educación, impartida en un total de 36 másteres, con una duración entre 2 y 6 créditos, siendo lo más habitual 4 créditos.

Otras materias en el módulo genérico son Organización y gestión de centros y Tecnología de la información y comunicación aplicada a la docencia y al aprendizaje (UMH) con 4,5 créditos y la UPM ( 3 créditos) cada una de ellas. La materia Formación psicopedagógica y social (UAB) con 15 créditos y en el máster interuniversitario con 12 créditos. Orientación profesional y ciudadanía (URV) de 3 créditos. En el máster de la Universidad Jaime I se imparte SIM-Instituto ( 2 créditos) y en el Master de la Universidad Pompeu Fabra- Oberta de Catalunya la asignatura Desarrollo, aprendizaje y educación (5 créditos), dentro del módulo de Fundamentos de la Educación Secundaria.

Por último, el plan de estudios de algunos másteres publicado en el $\mathrm{BOE}$ recoge sólo las asignaturas sin especificar la materia o el módulo. En concreto en el máster de la Universidad de Vigo en el plan de estudios contempla las asignaturas Orientación y función tutorial (3.5 créditos), Desarrollo psicológico del aprendizaje en la Educación Secundaria (4.5 créditos), Diseño curricular y organización de centros educativos (4,5 créditos), Sistema educativo y educación en valores (3,5 créditos). En el máster de la Universidad de Santiago de Compostela sucede algo similar, siendo las asignaturas Didáctica, currículo y organización escolar (4,5 créditos), La función tutorial y la orientación académica (2,5 créditos), Educación y lenguas en Galicia (2 créditos), Desarrollo psicológico y aprendizaje escolar (3,5 créditos) y Educación, sociedad y política educativa ( 3,5 créditos). Por último, en el máster de la Universidad de Zaragoza se recogen las asignaturas sin especificar si el módulo es genérico o específico ni la materia. En el caso del Máster de la Universidad de Girona el plan de estudios no aparece publicado en el BOE según información del RUCT.

Tabla 3.

Módulo genérico: Materias comunes y número de créditos por máster. Aprendizaje y desarrollo de la personalidad

3 créditos: Politécnica de Madrid y Rey Juan Carlos. 4 créditos: A Coruña, Alcalá, Almería, Autónoma de Madrid, Cádiz, Cantabria, Complutense de Madrid, Córdoba (solo en la especialidad de Orientación Educativa), Granada, Huelva, Jaén, Málaga, Pablo Olavide, Politécnica de Catalunya, Rovira i Virgili, Sevilla, Valencia y Valladolid. 4,5 créditos: La Rioja y Miguel Hernández. 5 créditos: Barcelona, Burgos, Castilla La Mancha, León, Lleida, Oviedo, Pública de Navarra y UNED. 6 créditos: Alicante, Extremadura, Jaime I, Murcia, País Vasco y Salamanca. 


\section{Procesos y contextos educativos}

3 créditos: Murcia y Politécnica de Madrid. 4 créditos: Alcalá, Almería, Cádiz, Complutense de Madrid, Córdoba, Granada, Huelva, Jaén, Málaga, Pablo Olavide, Politécnica de Catalunya, Rovira i Virgili, Sevilla y Valladolid. 4,5 créditos: La Rioja y Miguel Hernández. 5 créditos: Alicante, Barcelona, Burgos, Castilla La Mancha, Extremadura, León, Lleida, Pompeu Fabra-Oberta de Catalunya y UNED. 6 créditos: Autónoma de Madrid, Jaime I, País Vasco, Pública de Navarra, Rey Juan Carlos y Salamanca (estos créditos se comparten con la materia Sociedad, Familia y Educación como una única asignatura). 6,5 créditos: A Coruña. 7 créditos: Cantabria y Oviedo. 8 créditos: Valencia

\section{Sociedad, familia y educación}

2 créditos: Castilla La Mancha y León. 3 créditos: Alicante, Oviedo, Politécnica de Madrid, Pública de Navarra, Rey Juan Carlos y Salamanca. 4 créditos: Alcalá, Almería, Autónoma de Madrid, Burgos, Cádiz, Cantabria, Complutense de Madrid, Córdoba, Granada, Huelva, Jaén, Málaga, Pablo Olavide, Politécnica de Catalunya, Rovira i Virgili, Sevilla, Valencia y Valladolid. 4,5 créditos: La Rioja y Miguel Hernández. 5 créditos: Barcelona, Lleida, Pompeu Fabra-Oberta de Catalunya y UNED. 5,5 créditos: A Coruña. 6 créditos: Jaime I, Murcia y País Vasco.

En relación al módulo específico, tres grandes materias aparecen recogidas en la mayoría de los másteres. Éstas son, (ver tabla 4), Complementos de formación, recogida en 37 másteres con una duración entre 4 y 15 créditos, siendo lo más habitual una duración de 6 créditos. La materia Aprendizaje y enseñanza de la materia (Didáctica), impartida en 40 másteres con una extensión entre 8 y 24, siendo lo más habitual una duración de 12 créditos. La materia Innovación docente e investigación educativa en la especialidad, impartida en 39 másteres con una duración entre 3 y 12 créditos, siendo lo más frecuente una duración de 6 créditos.

En el máster (UAB) la materia se denomina Especialidad y su didáctica, con una duración de 27 créditos. En la (UB) el módulo específico tiene 25 créditos. Las materias, son las mismas que se recogen en la tabla 4, y los créditos varían en función de la especialidad. En el caso de la Politécnica de Madrid se especifican las asignaturas pero no la materia a la que pertenece cada una de ellas.

Tabla 4.

\section{Módulo específico: Materias impartidas y número de créditos por máster}

\section{Complementos de formación}

4 créditos: Alcalá. 5 créditos: Pompeu Fabra-Oberta de Catalunya. 6 créditos: A Coruña (6- 8 dependiendo de la especialidad), Almería, Cádiz, Castilla La Mancha, Córdoba, Granada, Huelva, Jaén, La Rioja, Málaga, Pablo Olavide, Rey Juan Carlos, Rovira i Virgili, Salamanca, Sevilla y Valencia. 7 créditos: Politècnica de Catalunya. 8 créditos: Jaime I, Lleida, Murcia, Oviedo, Santiago de Compostela y Vigo. 9 créditos: Alicante, Cantabria y País Vasco. 10 créditos: Autónoma de Madrid, Burgos, UNED, Máster interuniversitario (UAB, UB, UOC, UPC y UPF). 12 créditos: Extremadura, León (12 a 16 créditos) y Pública de Navarra. 14 créditos: Valladolid. 15 créditos: Complutense de Madrid.

8 créditos: Jaime I. 9 créditos: Cantabria, País Vasco y Pública de Navarra. 10 créditos: Complutense de Madrid, Pompeu Fabra-Oberta de Catalunya y Rovira i Virgili. 11 créditos: Oviedo (11 obligatorios/3 optativos). 12 créditos: A Coruña, Almería, Autónoma de Barcelona, Autónoma de Madrid, Cádiz, Castilla 
La Mancha, Córdoba, Extremadura, Granada, Huelva, Jaén, Lleida, Málaga, Pablo Olavide, Rey Juan Carlos, Santiago de Compostela, Sevilla, UNED, Valladolid, Vigo, Máster interuniversitario (UAB, UB, UOC, UPC y UPF) y Miguel Hernández (se denomina didáctica y aproximación al currículum). 14 créditos: Burgos. 15 créditos: Alicante, La Rioja, Murcia, Politécnica de Catalunya y Salamanca. 16 créditos: Alcalá, León y Valencia. 24 créditos: Vigo (24 todos optativos)

\section{Innovación docente e investigación educativa en la especialidad}

3 créditos: Santiago de Compostela. 4 créditos: Alcalá, Autónoma de Madrid, Burgos, Murcia y Oviedo. 5 créditos: Complutense de Madrid, Lleida, Rovira i Virgili y UNED. 6 créditos: A Coruña 6 y 8 dependiendo de la especialidad), Alicante, Almería, Autónoma de Barcelona, Cádiz, Cantabria, Castilla La Mancha, Córdoba, Extremadura, Granada, Huelva, Jaén, La Rioja, Málaga, País Vasco, Pablo Olavide, Politécnica de Catalunya, Pública de Navarra, Rey Juan Carlos, Salamanca, Sevilla, Valencia, Valladolid, Vigo y Máster interuniversitario (UAB, UB, UOC, UPC y UPF). 8 créditos: Jaime I y León. 10 créditos: Pompeu Fabra-Oberta de Catalunya. 12 créditos: Miguel Hernández (se denomina Innovación docente e Iniciación a la investigación educativa y atención al alumnado con necesidades educativas especiales).

\section{Conclusiones}

La oferta ofrecida por la Universidad pública española en materia de Formación del Profesorado en Educación Secundaria, Bachillerato, Formación Profesional y Enseñanzas de Idiomas, Artísticas y Deportivas es amplia si se tiene en consideración que se imparte en 43 universidades repartidas por todo el territorio nacional y el número de plazas ofertadas. Si bien, se puede decir que existe una clara descompensación en relación a la formación en Educación Secundaria y Bachillerato con respecto a la Formación Profesional y las Enseñanzas Especiales, si se tiene en cuenta que solo en 27 másteres se imparte la formación en materia de Procesos industriales, Hostelería y turismo, Construcciones civiles, Intervención sociocomunitaria, Biosanitaria o Servicios primario y secundario. Esta discrepancia puede ser fácilmente explicable atendiendo a la obligatoriedad de la Enseñanza Secundaria.

En este sentido cabe reflexionar acerca de la Formación Profesional, más si se tiene en consideración la relevancia presente y futura que este tipo de formación puede tener en las políticas educativas y laborales, si se tiene en cuenta el número de profesionales que demanda el mercado laboral, formación que cuenta con una alta inserción laboral y la incorporación de un mayor número de estudiantado a este tipo de formación. Si se tiene en consideración el número de estudiantes matriculados en España por nivel educativo en el curso académico 2009-2010, año en el que se implantan la mayoría de los másteres analizados, y el curso académico 2017-2018, según datos del Ministerio de Educación (2019), mientras que en la Educación Secundaria Obligatoria se produce un aumento de 136.891 estudiantes y en Bachillerato de 25.427 entre un año y otro, en la Formación Profesional el aumento es de 192.543 estudiantes.

Por otro lado, la oferta de especialidades en el caso de la Formación Profesional no atiende la totalidad de las 26 familias profesionales existentes, y en otros casos es escaso el número de másteres que 
atienden de forma específica esta formación, como por ejemplo el caso de la familia Edificación y Obra Civil que sólo se oferta en el máster de la Universidad de Alicante. Son las familias relacionadas con Procesos Industriales, Sanidad, Administración y Gestión y los Servicios Socioculturales y a la Comunidad los más representados, frente a otras familias profesionales como Agraria, Artes y Artesanía, Madera, Mueble y Corcho o Seguridad.

En el caso de la Educación Secundaria y Bachillerato son las especialidades de Filosofia o Lenguas Clásicas las menos representadas. En el caso de los idiomas, es la especialidad de inglés la más representada frente a otros idiomas.

La formación ofertada presenta una serie de diferencias según las variables estudiadas, como el nombre del Máster, no incluyendo los diferentes niveles o enseñanzas que luego sí se imparten en el master. En este sentido cabría normalizar los títulos de los másteres recogiendo todos los niveles educativos que se imparten.

Otras diferencias están relacionadas con los créditos del módulo genérico y los créditos del módulo específico, variando de un máster a otro. En relación a la optatividad, entendida como créditos de libre elección fuera de las especialidades, solo 11 másteres ofrecen estos créditos, variando tanto el número de créditos como la oferta. Con respecto al módulo específico la Orden ECI/3858/2007 apunta a un mínimo de 24 créditos para este módulo, en este sentido diferentes másteres se encontrarian por debajo de este mínimo, como son el de la Universidad de Oviedo con 23 créditos, la Universidad Politécnica de Madrid con 18 créditos y la Universitat Rovira i Virgili con 20 créditos. En los dos primeros casos cabría analizar si la presencia de créditos optativos van asociados al módulo específico, si bien en el último caso no existe optatividad.

A modo de conclusión, tras obtener una visión general de los másteres relacionados con la formación del profesorado en Educación Secundaria y otros ciclos educativos, después de 10 años de su puesta en marcha, cabe decir que todos ellos siguen una estructura muy similar, tal y como así recoge la ley que regula esta formación, si bien existen diferencias principalmente en el número de créditos para cada una de las materias.

Resulta necesario abordar el tema de la formación profesional, no solo en cuanto al número de másteres que ofrecen formación en este ámbito sino también con respecto a las diferentes familias profesionales. Sería conveniente la inclusión de la formación de adultos, más si se tiene en consideración el aumento de edad del alumnado que puede haber en la Formación Profesional así como la incorporación de un estudiantado que se reengancha al estudio, ya que la formación general aborda en gran medida el periodo evolutivo de la adolescencia.

Después de 10 años de implantación del Máster en Formación del Profesorado cabe necesaria la organización de jornadas de trabajo o congresos científicos acerca de las posibilidades de mejora y de cambio 
en la formación del profesorado, con vistas a posibilitar una formación acorde a las características cambiantes del alumnado y de los centros educativos.

Tal y como recoge la Conferencia de Decanos/as de Educación (2017) resulta necesaria concebir la profesión docente como un continuo frente al actual sistema fragmentado en etapas, aplicar otros criterios, no solo la nota media, a la hora de poder cursar el Master de Secundaria, revisar las plazas ofertadas según la demanda de empleo, aumentar la experimentalidad en la formación o rediseñar los planes de estudios desde un enfoque de competencias y de perfiles profesionales docentes.

Esteve (2009) plantea como principales retos de la formación del profesorado a través del máster el desarrollo de una identidad profesional, saber atender las demandas que se producen en la interacción dentro del aula, la construcción de un aprendizaje significativo y la capacidad del profesorado para que el grupo clase sea capaz de abordar el aprendizaje de una forma efectiva. Desde esta perspectiva cobra gran relevancia la formación psicopedagógica, que permita conocer las interacciones dentro del aula, las necesidades y problemáticas, la aplicación de estrategias de intervención, partir de lo que saben los alumnos-as, asumiendo que no todos parten del mismo punto en cuanto a conocimientos, metodologias de aprendizaje y situaciones sociofamiliares que pueden interferir en el aprendizaje.

Escudero (2009) habla de un master más centrado en competencias que en ámbitos de la profesión de profesor, organizado desde una perspectiva disciplinar, fragmentado, no habiendo en algunos casos correspondencia entre los módulos del mismo y las competencias, con poca relevancia del módulo de Aprendizaje y Desarrollo, y que no recoge toda una serie de valores relacionados con la profesión, como son tomar conciencia de que todos los estudiantes pueden aprender, responsabilidad y cuidado de los alumnos, consideración y respeto hacia la diversidad, compromiso y dedicación, espíritu de equipo e implicación en el aprendizaje continuo y la búsqueda de la excelencia profesional.

Según Manso y Valle (2013), en el caso de España el porcentaje en formación didáctico-pedagógica es del 20\% de los créditos del máster, siendo el porcentaje minimo en esta formación entre el 20 y el $25 \%$ en la formación del profesorado en otros países europeos. Estos autores apuntan a una necesidad de aumentar este tipo de formación en la formación inicial del profesorado, mejorar los procesos de selección y una mayor práctica docente.

Como bien apunta González-Sanmamed (2009) resulta necesario cuidar y mejorar la formación de los profesores, a ello hay que añadir la necesidad de revisar esta formación en el eje tiempo espacio, de tal forma que después de diez años de puesta en marcha del máster y de cambios importantes a nivel social, de los alumnos, de los contenidos y metodologias, entre otros aspectos, resulta necesario actualizar, y si 
fuese necesario mejorar, la formación del profesorado.

Prieto, Díaz y Santiago (2014) consideran relevante la inclusión de metodologias activas, inductivas, colaborativas y con alta participación del alumnado, siendo éste responsable de su propio aprendizaje. En este sentido, Martín y Santiago (2016) en un estudio realizado con estudiantes del master de formación del profesorado en secundaria, concluyen la necesidad de incluir en esta formación el modelo Flipped Learning.

Este estudio presenta diferentes limitaciones, la principal es que sólo se ha tenido en cuenta los másteres oficiales impartidos por Universidades públicas, no abordando el análisis de los ofertados por Universidades privadas, si bien cabe decir que el presente trabajo ofrece una visión general del panorama nacional de la formación del profesorado en Secundaria y Formación Profesional después de diez años de implantación.

\section{Referencias}

Coelho, E., Oller, J. y Sierra, J. M. (2011). Repensando la formación inicial del profesorado para abordar el tratamiento a la diversidad cultural y lingüística en el aula, @tic. Revista d'Innovació Educativa, 7, 52-61.

Conferencia de Decanos/as de Educación (2017). Conclusiones jornada monogràfica: El modelo de acceso a la profesión docente actual vs "MIR" educativo. Recuperado de http://reunid.eu/wpcontent/uploads/2018/02/2017_09_28_DocumentoConferencia-Educacion-sobre-Formacion-de-Profesorado.pdf

Escudero, J. M. (2009). La formación del profesorado de Educación Secundaria: contenidos y aprendizajes docentes. Revista de Educación, 350, 79-103.

Esteve, J. M. (2001). El profesorado de secundaria: hacia un nuevo perfil profesional para enfrentar los problemas de la educación contemporánea. Fuentes: revista de la Facultad de ciencias de la Educación, 3, 15-42.

Esteve, J. M. (2009). La formación de profesores: bases teóricas para el desarrollo de programas de formación inicial. Revista de Educación, 350, 15-29.

González-Sanmamed, M. (2009). Una nueva oportunidad para la formación inicial del profesorado de Educación Secundaria. Revista de Educación, 350, 57-78.

Manso, J. y Valle, J. M. (2013). La formación inicial del profesorado de 
secundaria en la Unión Europea. Revista Española de Educación Comparada, 22, 165-184.

Martín, D. y Santiago, R. (2016). Flipped Learning en la formación del profesorado de secundaria y bachillerato. Formación para el cambio. Contextos Educativos, Extraordinario, 1, 117-134. Doi: 10.18172/con.2854.

Ministerio de Educación (2019). Enseñanza no universitaria. Alumnado matriculado. Recuperado de http: / /www.educacionyfp.gob.es/servicios-al-ciudadanomecd/estadisticas/educacion/nouniversitaria/alumnado/matriculado.html

Prieto, A., Diaz, D. y Santiago, R. (2014). Metodologias inductivas: El desafio de enseñar mediante el cuestionamiento y los retos. Barcelona: Digital-text.

Rebolledo, T. (2015). La formación inicial del profesorado en Educación primaria y secundaria en Alemania, España, Finlandia, Francia y Reino Unido. Estudio comparado. Revista Española de Educación Comparada, 25, 129-148. Doi: 10.5944/reec.25.2015.14787

Vilches, A. y Gil-Pérez, D. (2010). Máster de formación inicial del profesorado de Enseñanza Secundaria. Algunos análisis y propuestas. Revista Eureka de Enseñanza y Divulgación Científica, 7(3), 661-666. 
